Onkologe 2021 $27: 282-286$

https://doi.org/10.1007/s00761-021-00901-2

Angenommen: 13. Januar 2021

Online publiziert: 25. Januar 2021

(c) Springer Medizin Verlag $\mathrm{GmbH}$, ein Teil von Springer Nature 2021

R. Bremen ${ }^{1} \cdot$ A. Petermann-Meyer ${ }^{2} \cdot$ N. Ernstmann ${ }^{3} \cdot$ E. Jost $^{1} \cdot$ J. Panse ${ }^{1} \cdot$

T. H. Brümmendorf'

${ }^{1}$ Klinik für Hämatologie, Onkologie, Hämostaseologie und Stammzelltransplantation, Medizinische Klinik IV, Centrum für Integrierte Onkologie (CIO-A), Medizinische Fakultät, Uniklinik RWTH Aachen, Aachen, Deutschland

${ }^{2}$ Klinik für Hämatologie, Onkologie, Hämostaseologie und Stammzelltransplantation, Sektion Psychoonkologie, Medizinische Klinik IV, Centrum für Integrierte Onkologie (CIO-A), Uniklinik der RWTH Aachen, Aachen, Deutschland

${ }^{3}$ Forschungsstelle für Gesundheitskommunikation und Versorgungsforschung, Klinik und Poliklinik für psychosomatische Medizin und Psychotherapie, Centrum für integrierte Onkologie (CIO-B), Universitätsklinikum Bonn, Bonn, Deutschland

\title{
Sollen Kinder onkologischer Patienten während der COVID- 19-Pandemie Kitas oder Schulen besuchen?
}

\section{COVID-19-Infektion für den erkrank- ten Elternteil aus einem Besuch der Kinder von Kitas und Schulen resultieren.}

Etwa $14 \%$ aller Krebspatienten haben minderjährige Kinder [5]. Jährlich erkranken Expertenschätzungen zufolge in Deutschland rund 37.000 Eltern minderjähriger Kinder neu an Krebs, betroffen sind damit ca. 50.000 Kinder [9]. Eine elterliche Krebserkrankung ist in emotionaler wie auch organisatorischer Hinsicht grundsätzlich eine große Herausforderung [2]. Die Folgen der Erkrankung betreffen die gesamte Familie [3]. Das Risiko für die Entwicklung psychischer Probleme und affektiver Symptome ist bei den Kindern und dem gesunden Elternteil im weiteren Verlauf deutlich erhöht [7, 8]. Gerade das Aufrechterhalten von sicherheitsspendenden Alltagsabläufen ist neben einer möglichst offenen Kommunikation ein bekannter protektiver Faktor [1].

Das vom Centrum für Integrierte Onkologie Aachen (CIOA) initiierte multizentrische Forschungs- und Versorgungsprojekt „Familien-SCOUT" hat es sich zur Aufgabe gemacht, alle Familienmitglieder eines an Krebs erkrankten Elternteils auf organisatorischer, emo- tionaler und kommunikativer Ebene $\mathrm{zu}$ unterstützen und eine daraus resultierende etwaige Belastungsreduktion systematisch zu evaluieren.

Die Fragestellung, inwieweit während der COVID-19-Pandemie gewohnte Alltagsstrukturen weiterbestehen und Betreuungsangebote in Anspruch genommen werden können, ist für Eltern, Kinder und auch für behandelnde Onkologen ein höchst relevantes Thema. Dazu gehört auch und in besonderem Maße der Besuch von Kitas bzw. Schulen durch die Kinder der Betroffenen.

\section{Befragung von onkologischen Experten}

Wer kann diese Entscheidung für die Kinder der Betroffenen treffen? Welche Kriterien sind bei der Risikoeinschätzung zu berücksichtigen, und existieren allgemeine Empfehlungen?

Da mangels vorhandener Daten keine belastbare Evidenz für eine solche Empfehlung existiert, wurden im Sommer 2020 noch vor Beginn des zweiten Lockdowns die Mitglieder des Lenkungskreises des deutschen Netzwerks Onkologischer Spitzenzentren der Deutschen Krebshilfe (DKH), dem die Direktoren von 18 Onkologischen Spitzen-

Die Autoren R. Bremen und A. Petermann-Meye teilen sich die Erstautorschaft. 
Wer soll entscheiden, ob die Kinder die Kita oder Schule besuchen

dürfen?

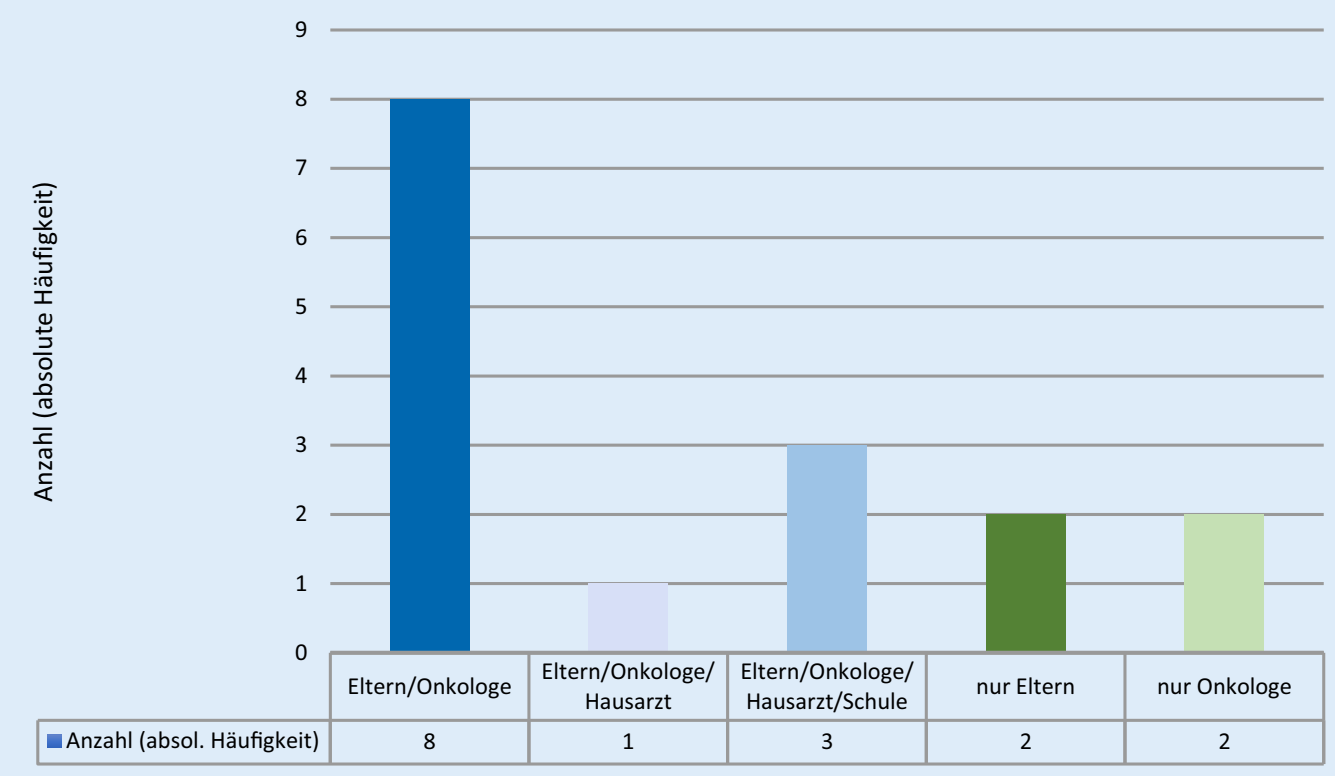

Abb. $1 \varangle$ Häufigkeit der ausgewählten Entscheidungsträger. Aufgrund möglicher Mehrfachnennungen auch Häufigkeit der ausgewählten Kombinationen. $(n=16 / 17 \mathrm{Be}$ fragte, 34 Nennungen)

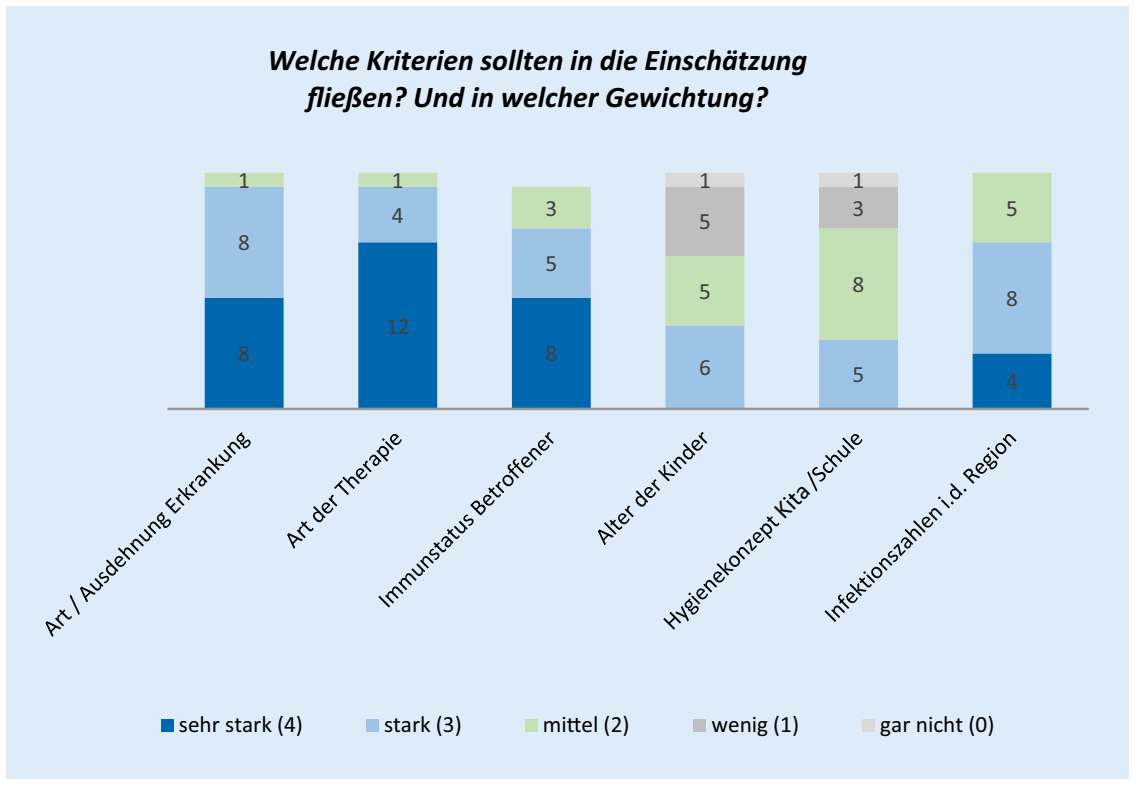

Abb. 2 \ Darstellung der Auswahl und Gewichtung der für den Entscheidungsprozess relevanten Kriterien. Oben dargestellte Kriterien waren vorgegeben und auf einer 5-stufigen Likert-Skala zu gewichten ( $n=17$, Kriterium Immunstatus Betroffener $n=16$ )

zentren angehören, anhand eines eigens entwickelten Fragebogens um ihre Expertenmeinung gebeten.

Der Fragebogen beinhaltet Fragen - zur Häufigkeit und zur Relevanz der Fragestellung im klinischen Alltag,
- zu den der Entscheidung zugrunde liegenden Kriterien und ihrer Gewichtung,

- zur Verantwortung für die Entscheidungsfindung und

- zu einer möglichen allgemeinen Empfehlung.
Insgesamt nahmen 15 Zentren an der Befragung teil, 17 unabhängige Expertenmeinungen sind in die Auswertung eingeflossen. Die Mehrheit der Befragten gab an, der Frage besorgter Eltern nach dem Besuch ihrer Kinder von Kitas und Schulen und dem Risiko einer möglichen COVID-19-Infektion für sie als Patienten im klinischen Setting ca. einmal pro Woche zu begegnen. Die Frage ist laut 14 Experten entsprechend von Relevanz.

Auf die Frage, wer die Entscheidung zum Kita- und Schulbesuch der Kinder fällen soll, geben die Befragten in gleicher Anzahl die Eltern/Familie und behandelnde Onkologen als am besten geeignete Entscheidungsträger an. Präferenziell wird eine partizipative Entscheidungsfindung (Mehrfachnennung) zwischen Onkologen und Familien empfohlen (• Abb. 1).

Risikokriterien, die bei der Entscheidungsfindung vorrangig berücksichtigt werden sollten, sind die Art/Ausdehnung der Erkrankung und die Art der Therapie bzw. der daraus resultierende Immunstatus der Betroffenen. Auch das regionale Infektionsgeschehen wird als relevantes Kriterium gewertet (• Abb. 2).

Keiner der Befragten sprach sich dafür aus, dass die Kinder Schule und Kita ge- 
Hier steht eine Anzeige.

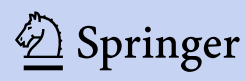


Falls Sie eine allgemeine Empfehlung geben sollten, was würden Sie z.Zt. am ehesten empfehlen?

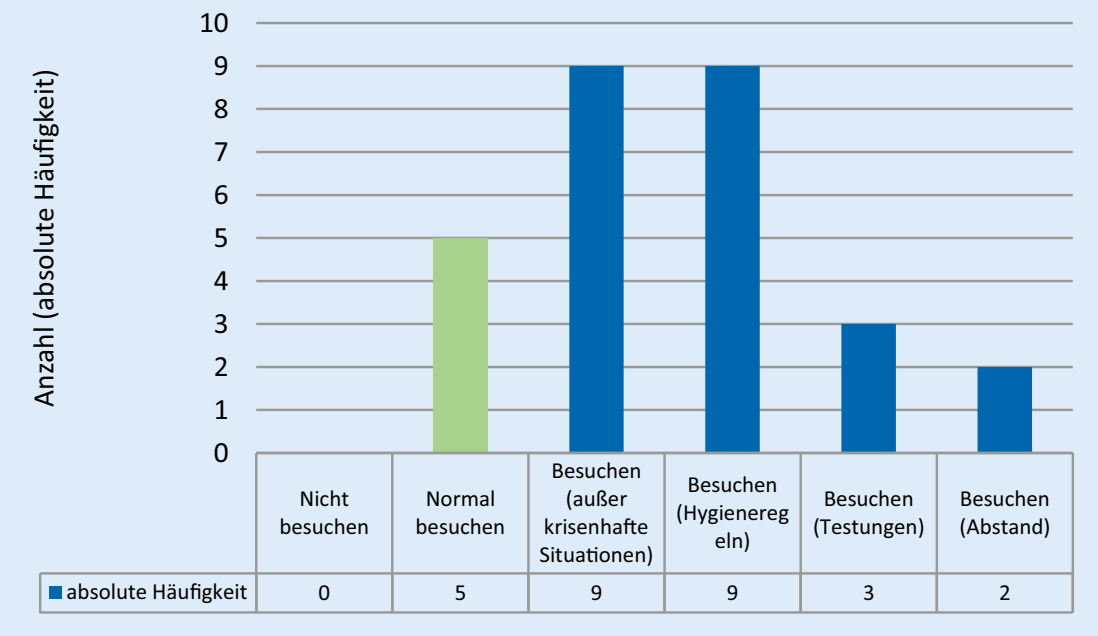

Abb. $3 \Delta$ Abfrage einer allgemeinen Empfehlung zum Schulbesuch. Mehrfachnennungen möglich, Antwortmöglichkeiten nicht hierarchisch sortiert, z. T. Kombination von "normal besuchen" mit oben angeführten Einschränkungen, Nennungen $(n=28)$, Befragte $(n=17)$

nerell nicht mehr besuchen sollen, wenn anhand der oben genannten Kriterien keine deutlich erhöhte Gefährdung für den betroffenen Elternteil besteht. Allgemein empfahl die Mehrheit der Befragten, dass Kinder von an Krebs erkrankten Eltern Kitas und Schulen unter strengerer Einhaltung der Hygieneregeln besuchen und allenfalls in krisenhaften Situationen temporär den Schulbesuch aussetzen (• Abb. 3).

\section{Implikationen für die Praxis}

Eine allgemeingültige Empfehlung für alle betroffenen Familien war aufgrund der mangelnden Evidenz und der diversen individuellen onkologischen, psychosozialen und regionalen Situationen nicht möglich. Nach Ansicht des Expertengremiums der Onkologischen Spitzenzentren soll Kindern von an Krebs erkrankten Eltern der Schulbesuch jedoch grundsätzlich ermöglicht werden. Im begründeten Einzelfall einer zumindest transient deutlich erhöhten Gefährdung für den betroffenen Elternteil kann der Schulbesuch unter Umständen kurzfristig ausgesetzt werden. Zum Zeitpunkt der Erhebung war die diesbezügliche wissenschaftlich fundierte Datenlage bestenfalls spärlich. Aktuell zeigen prospektive Auswertungen Hinweise auf mögliche spezifische Risikogruppen, z. B. hämatologisch-onkologische Systemerkrankungen mit Beeinträchtigung des ImmunsysImmunabwehr im Hinblick auf eine adäquate Reaktion gegen eine mögliche Virusinfektion wie auch perspektivisch die Immunreaktion auf einen potenziell verfügbaren Impfstoff direkt beinträchtigen [6]. Neben den medizinischen Aspekten gilt es zudem, das regionale und aktuelle Infektionsgeschehen zu berücksichtigen, um eine Abwägung, wann ein erhöhtes Risiko besteht, zu vervollständigen.

Aufgrund der stetig neuen Erkenntnislage und Komplexität der klinischen Bewertung sowie der Schwierigkeit, daraus ein individuelles Risiko abzuleiten, sollten betroffene Eltern die Entscheidung unter Berücksichtigung ihrer onkologischen Gesamtsituation gemeinsam mit den behandelnden Onkologen treffen und im Falle einer geänderten Faktenlage auch kurzfristig anpassen können. Eltern und Onkologen wird damit eine gemeinsame Verantwortung zugesprochen. Die Entscheidung soll partizipativ getroffen werden, unter Umständen auch in Abstimmung mit dem Hausarzt und der Schule. Betroffenen Familien kann das insofern Entlastung verschaffen, als die Entscheidung unter Berücksichtigung der individuellen medizinischen Situatems und/oder Therapieformen, die die tion nicht allein getroffen werden muss und sie und ihre Kinder sich für etwaige Konsequenzen, wie eine im Verlauf möglicherweise auftretende COVID-19Infektion, nicht schuldig fühlen müssen. Da mehrheitlich als Entscheidungskriterium die Art/Ausdehnung der Erkrankung und die Art der Therapie sowie der daraus resultierende Immunstatus des Betroffenen benannt wurden, erscheint hier die Befähigung betroffener Eltern zu einer informierten Entscheidungsfindung im Austausch mit den behandelnden Onkologen und durch fundierte Empfehlungen essenziell. Die behandelnden Onkologen sind in ihrer Rolle als medizinische Experten maßgeblich daran beteiligt, Familien notwendiges Fachwissen zu vermitteln und Auskunft über onkologische und behandlungsspezifische Faktoren sowie ihre potenzielle Auswirkung auf das Risiko einer COVID-19-Infektion zu geben.

\section{Korrespondenzadresse}

\section{Dr. med. A. Petermann-Meyer}

Klinik für Hämatologie, Onkologie, Hämostaseologie und Stammzelltransplantation, Sektion Psychoonkologie, Medizinische Klinik IV, Centrum für Integrierte Onkologie (CIO-A), Uniklinik der RWTH Aachen

Pauwelsstraße 30, 52074 Aachen, Deutschland apetermann-meyer@ukaachen.de

Danksagung. Ein herzlicher Dank gilt allen weiteren Mitwirkenden: Prof. Dr. med. Peter Albers, Prof. Dr. med. Hana Algül, Prof. Dr. med. Ralf Bargou, Prof. Dr. med. Carsten Bokemeyer, Prof. Dr. med. Martin Bornhäuser, Prof. Dr. med. Christian H. Brandts, Prof. Dr. med. Peter Brossart, Prof. Dr. med. Stefan Fröhling, Prof. Dr. med. Michael Hallek, Prof. Dr. med. Volker Heinemann, Prof. Dr. med. Ulrich Keilholz, Prof. Dr. med. Thomas Kindler, Prof. Dr. med. Florian Lordick, Prof. Dr. med. Christoph Peters, Prof. Dr. med. Daniel Zips, Prof. Dr. med. Olaf Ortmann, Gerd Nettekoven

\section{Einhaltung ethischer Richtlinien}

Interessenkonflikt. R. Bremen, A. Petermann-Meyer, N. Ernstmann, E. Jost, J. Panse und T.H. Brümmendorf geben an, dass kein Interessenkonflikt besteht.

Für diesen Beitrag wurden von den Autoren keine Studien an Menschen oder Tieren durchgeführt. Für die aufgeführten Studien gelten die jeweils dort angegebenen ethischen Richtlinien. 


\section{Was Patienten fragen}

\section{Literatur}

1. Buchbinder M, Longhofer J, McCue K (2009) Family routines and rituals when a parent has cancer. Fam Syst Health 27(3):213

2. Inhestern L, Haller AC, Wlodarczyk O, Bergelt C (2016) Psychosocial interventions for families with parental cancer and barriers and facilitators to implementation and use - a systematic review. PLOSONE 11(6): 156967

3. Inhestern L, Bultmann JC, Beierlein V, Möller B, Romer G, Koch U, Bergelt C (2017) Elterliche Sorgen und psychische Belastung bei krebskranken Eltern mit minderjährigen und jungerwachsenen Kindern. Psychother Psychosom Med Psychol 67(07):279-287

4. Košir U, Loades M, Wild J, Wiedemann M, Krajnc A Roškar S, Bowes L (2020) The impact of COVID-19 on the cancer care of adolescents and young adults and their well-being: results from an online survey conducted in the early stages of the pandemic Cancer 126(19):4414-4422

5. Krauel K, Simon A, Krause-Hebecker N, Czimbalmos A, Bottomley A, Flechtner H (2012) When a parent has cancer: challenges to patients, their families and health providers. Expert Rev Pharmacoeconom Outcomes Res 12(6):795-808

6. Lee LY, Cazier JB, Starkey T, Briggs SE, Arnold R, Bisht V, Curley HMetal (2020) COVID-19 prevalence and mortality in patients with cancer and the effect of primary tumour subtype and patient demographics: a prospective cohort study. Lancet Oncol 21(10):1309-1316

7. Möller B, Barkmann C, Krattenmacher T, Kühne F, Bergelt C, Beierlein V, Romer G et al (2014) Children of cancer patients: Prevalence and predictors of emotional and behavioral problems. Cancer 120(15):2361-2370

8. Nakaya N, Saito-Nakaya K, Bidstrup PE, Dalton SO, Frederiksen K, Steding-Jessen M, Johansen C et al (2010) Increased risk of severe depression in male partners of women with breast cancer. Cancer 116(23):5527-5534

9. Robert Koch Instititut (2019) https:// www.krebsdaten.de/Krebs/DE/Content/ Publikationen/Kurzbeitraege/Archiv2019/ 2019_3_Kurzbeitraege_Krebskranke_Eltern.html

\section{Hier steht eine Anzeige.}

\section{算 Springer}

